ifosfamide in 2 pts (12\%) and other regimens in 3 pts (18\%). Pts treated with ibrutinib received $560 \mathrm{mg}$ p.o. daily. Intratechal CT was added in 5 pts (31\%) and 7 pts (47\%) in standard and ibrutinib cohort. Radiotherapy was delivered to 3 pts, all in the standard cohort, in one case as consolidation and in 2 cases as salvage. With a median followup of 10.4 months, the 1-year PFS and OS of the entire study population are $24 \%$ and $46 \%$. A statistically significant difference in 1-year PFS was observed in favor of ibrutinib versus standard CT ( $49 \%$ vs $6 \%, p=0.044)$. The difference in 1-year OS in favor of ibrutinib versus standard CT did not reach statistical significance $(57 \%$ vs $37 \%$, $\mathrm{p}=0.097$ ). In the standard cohort only one pt is alive after allogeneic transplantation.

Conclusion: In this study, ibrutinib monotherapy appears to be effective for CNS-MCL; with the usual limitations of a retrospective analysis, our data show a benefit in PFS for CNS-MCL pts treated with ibrutinib in comparison to standard chemoimmunotherapy.

Keywords: ibrutinib; mantle cell lymphoma (MCL); salvage treatment. Disclosures: Rusconi, C: Research Funding: Celgene, Takeda (advisory board), Roche, Celgene; Other Remuneration: Takeda, Roche.

\title{
191 \\ UPDATED SAFETY AND EFFICACY DATA IN THE PHASE 1 TRIAL OF PATIENTS WITH MANTLE CELL LYMPHOMA (MCL) TREATED WITH BRUTON TYROSINE KINASE (BTK) INHIBITOR ZANUBRUTINIB (BGB-3111)
}

\section{$\underline{\text { C.S. Tam }}^{1}$ | M. Wang ${ }^{2}$ | D. Simpson ${ }^{3}$ | S. Opat ${ }^{4}$ |

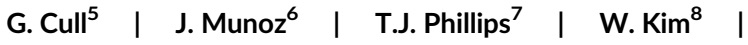

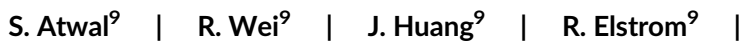 J. Trotman ${ }^{10}$ \\ ${ }^{1}$ Department of Haematology, Peter MacCallum Cancer Centre, St. Vincent's Hospital, University of Melbourne, Melbourne, Victoria, Australia; ${ }^{2}$ Department of Lymphoma \& Myeloma, Division of Cancer Medicine, MD Anderson Cancer Center, Houston, TX, United States; ${ }^{3}$ Waitemata DHB Haematology Service, North Shore Hospital, Auckland, New Zealand; ${ }^{4}$ Clinical Haematology, Monash Health, Monash University, Clayton, Victoria, Australia; ${ }^{5}$ Department of Haematology, Sir Charles Gairdner Hospital, University of Western Australia, Perth, WA, Australia; ${ }^{6}$ Hematology-Oncology, Banner MD Anderson Cancer Center, Gilbert, AZ, United States; ${ }^{7}$ Michigan Medicine Hematology Clinic, Rogel Cancer Center, University of Michigan, Ann Arbor, MI, United States; ${ }^{8}$ Samsung Medical Center, Sungkyunkwan University School of Medicine, Seoul, Republic of Korea; ${ }^{9}$ Research and Development Center, BeiGene (Beijing) Co., Ltd, Beijing, China; BeiGene USA, Inc., San Mateo, United States; ${ }^{10}$ Department of Haematology, Concord Repatriation Hospital, The University of Sydney, Concord, NSW, Australia}

Introduction: Zanubrutinib, an investigational BTK inhibitor, has demonstrated greater selectivity for BTK vs other TEC- and EGFR-family kinases in biochemical assays and shown favorable PK/PD properties in preclinical studies. In phase 1 testing, high plasma concentrations were achieved, resulting in complete and sustained 24-hour BTK inhibition in blood and lymph nodes in patients (pts) treated at $160 \mathrm{mg}$ twice daily (bid; Tam. Blood 2016;128:642). Here, we present updated safety and efficacy data from pts with MCL.

Methods: This is a global, phase 1 study investigating zanubrutinib in pts with B-cell malignancies with indication-specific expansion cohorts. In the expansion phase, enrolled pts received zanubrutinib $320 \mathrm{mg}$ daily or $160 \mathrm{mg}$ bid (the RP2D). Treatment emergent adverse events (TEAEs) were summarized according to $\mathrm{NCI}$ CTCAE v4.03 and responses were assessed by $\mathrm{CT}$ scans as per Lugano Classification (Cheson. J Clin Oncol 2014;32:3059).

Results: As of 16 Sep 2018, $48 \mathrm{MCL}$ pts were enrolled: 37 relapsed/refractory (R/R) and 11 treatment-naïve (TN) (Table). Of the 48 pts, 45 were evaluable for efficacy; 3 were not efficacy evaluable as they had not yet reached the first 12-week efficacy assessment. Median follow-up for efficacy evaluable pts was 16.0 mo (range, 1.6-38.2). Twenty-six pts discontinued treatment (16 due to progressive disease [PD]; 10 due to TEAEs including peripheral edema, small cell lung cancer, renal hematoma, ANCA-positive vasculitis with acute kidney injury, subdural hematoma, and myelodysplastic syndrome, pneumonia (2 pts), congestive cardiac failure, thalamic infarction). Five pts died due to TEAEs (1 pneumonia, 1 congestive cardiac failure, 1 thalamic infarction, and 2 sepsis/septic shock), none of which were assessed by investigator as related to zanubrutinib. Most common TEAEs of any cause $(\geq 15 \%$ of pts) included diarrhea (35\%), petechiae/purpura/contusion (31\%), upper respiratory tract infection (27\%), fatigue (25\%), constipation (21\%), rash $(19 \%)$, back pain (17\%), headache (17\%) and peripheral edema (17\%). Overall response rate (ORR) for TN, R/R and overall was $87.5 \%(7 / 8)$, $86.5 \%$ (32/37) and $86.7 \%$ (39/45) respectively (Table). Responses were based on computed tomography scans for most pts, as positronemission tomography was not required. Median progression-free survival was $15.4 \mathrm{mo}$ (Table).

Conclusions: Zanubrutinib monotherapy was shown to be well tolerated and highly active in pts with MCL, with high ORR and rate of CR. Keywords: BTK inhibitors; mantle cell lymphoma (MCL).

Disclosures: Tam, C: Honoraria: Beigene, Janssen, AbbVie, Novartis; Research Funding: Janssen and AbbVie. Wang, M: Consultant Advisory Role: Biolnvent; IO Biotech; Celgene; Juno Therapeutics; Janssen; Pharmacyclics; AstraZeneca; MoreHealth; Pulse BioSciences; AxImmune; Stock Ownership: MoreHealth; Honoraria: Janssen; Dava Oncology; OMI; PeerView Institute for Medical Education (PVI); Research Funding: Janssen; AstraZeneca; Acerta Pharma; Kite Pharma; Juno Therapeutics; BeiGene; Novartis; Celgene; Biolnvent; Karus; Oncternal; Amgen; Other Remuneration: Travel, Accommodations, Expenses: Janssen; AstraZeneca; Dava Oncology; OMI. Simpson, D: Honoraria: Celgene, Janssen, Abbvie, Roche; Research Funding: Amgen, Pharmacyclics, Acerta, Beigene, Celgene, BMS, Roche, Sanofi, GSK; Other Remuneration: Travel, Accommodations, Expenses: Janssen, Celgene. Opat, S: Consultant Advisory Role: Roche, Janssen, Abbvie, Celgene, Takeda, Merck, Gilead, Mundipharma; Honoraria: Roche, Janssen, Abbvie, Celgene, Takeda, Merck, Gilead, Mundipharma; Research Funding: BeiGene, Roche, Janssen, 
TAB LE 1 Patient Characteristics, Safety, and Efficacy

\begin{tabular}{|c|c|c|c|}
\hline Patient characteristics & $N=48$ & & \\
\hline Median (range) age, $y$ & $71(42-90)$ & & \\
\hline \multicolumn{4}{|l|}{ ECOG PS, n (\%) } \\
\hline 0 & $20(41.7)$ & & \\
\hline 1 & $22(45.8)$ & & \\
\hline 2 & $6(12.5)$ & & \\
\hline \multicolumn{4}{|l|}{ Stage at study entry, n (\%) } \\
\hline Stage I & $3(6.3)$ & & \\
\hline Stage II & $1(2.1)$ & & \\
\hline Stage III & $4(8.3)$ & & \\
\hline Stage IV & $40(83.3)$ & & \\
\hline \multicolumn{4}{|l|}{ MIPI, n (\%) } \\
\hline Low risk & $12(25.0)$ & & \\
\hline Intermediate risk & $18(37.5)$ & & \\
\hline High risk & $18(37.5)$ & & \\
\hline \multicolumn{4}{|l|}{ Disease status, $\mathrm{n}(\%)$} \\
\hline Treatment-naïve & $11(22.9)$ & & \\
\hline Relapsed/refractory & $37(77.1)$ & & \\
\hline Median (range) no. of prior therapies & $1(1-4)$ & & \\
\hline Median (range) follow-up, mo & $15.1(0.6-38.2)$ & & \\
\hline Bulky disease >10 cm, n (\%) & $3(6.3)$ & & \\
\hline Safety, n (\%) & $N=48$ & & \\
\hline Any AE & $47(97.9)$ & & \\
\hline Grade $\geq 3 \mathrm{AEs}$ & $28(58.3)$ & & \\
\hline Serious AEs & $19(39.6)$ & & \\
\hline AEs leading to zanubrutinib discontinuation & $11(22.9)$ & & \\
\hline AEs leading to death & $5(10.4)$ & & \\
\hline \multicolumn{4}{|l|}{ Efficacy } \\
\hline Best response per investigator ( $n$ ) & $\mathrm{TN}(\mathrm{n}=8)$ & $R / R(n=37)$ & Overall $(n=45)$ \\
\hline Overall response rate, $\mathrm{n}(\%) ; 95 \% \mathrm{Cl}$ & $\begin{aligned} & 7(87.5) \\
& 47.3,99.7\end{aligned}$ & $\begin{array}{l}32 \text { (86.5); } \\
71.2,95.5\end{array}$ & $\begin{array}{l}39 \text { (86.7); } \\
73.2,94.9\end{array}$ \\
\hline Complete response, $\mathrm{n}(\%)$ & $3(37.5)$ & $11(29.7)$ & 14 (31.1) \\
\hline Partial response, n (\%) & $4(50.0)$ & $21(56.8)$ & $25(55.6)$ \\
\hline Stable disease, $\mathrm{n}(\%)$ & $0(0.0)$ & $2(5.4)$ & $2(4.4)$ \\
\hline Progressive disease, $\mathrm{n}(\%)$ & $1(12.5)$ & $3(8.1)$ & $4(8.9)$ \\
\hline Median follow up (min, max) & $8.6(1.6,25.0)$ & $17.1(1.9,38.2)$ & $16.0(1.6,38.2)$ \\
\hline Duration of response (mo) & $R / R(n=32)$ & & Overall $(n=39)$ \\
\hline Follow up, median $(\min , \max )^{a}$ & $14.7(0.0,28.2)$ & $14.3(0.0,28.2)$ & \\
\hline Median $(95 \% \mathrm{Cl})^{\mathrm{b}}$ & $14.7(10.6,18.5)$ & $14.7(10.6,18.5)$ & \\
\hline
\end{tabular}

Abbreviations: AE, adverse event; ECOG PS, Eastern Cooperative Oncology Group performance status; $\mathrm{Cl}$, confidence interval.

${ }^{a}$ Follow-up time is estimated by the reverse Kaplan-Meier method.

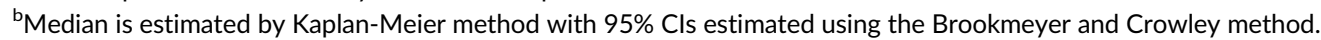

Abbvie, Takeda, Merck, Gilead, Epizyme. Cull, G: Research Funding: Beigene, Glycomimetics, Abbvie; Other Remuneration: Travel, Accommodations, Expenses: Amgen, Roche. Munoz, J: Consultant Advisory Role: Pharmacyclics LLC, Bayer, Gilead/Kite Pharma, Bristol-Myers Squibb, Janssen, Juno/Celgene; Other Remuneration: Speaker's Bureau: Kite Pharma, Gilead, Bayer, Pharmacyclics/Janssen, AstraZeneca. Phillips, T:
Consultant Advisory Role: Bayer, Gilead, Seattle Genetics, Genentech, Incyte, Pharmacyclics; Research Funding: Pharmacyclics, Abbvie. Kim, W: Research Funding: Roche, Takeda, Mundipharma, J\&J, Celltrion, Kyowa kirin, Donga. Atwal, S: Employment Leadership Position: BeiGene; Stock Ownership: BeiGene; Research Funding: BeiGene; Other Remuneration: Leadership: BeiGene. Wei, R: Employment Leadership Position: BeiGene; 
Stock Ownership: BeiGene. Huang, J: Employment Leadership Position: BeiGene; Stock Ownership: BeiGene. Elstrom, R: Employment Leadership Position: BeiGene; Stock Ownership: BeiGene. Trotman, J: Research Funding: PCYC, Roche, Janssen, Celgene, BeiGene.

\section{AGGRESSIVE LYMPHOMAS}

\section{2}

THE CLINICAL COURSE OF DIFFUSE LARGE B-CELL LYMPHOMA (DLBCL) OVER TIME: A MULTISTATE SURVIVAL ANALYSIS USING META-DATA FROM 13 FIRST-LINE RANDOMIZED TRIALS

\author{
Ç. Çăglayan $^{1}$ | J.G. Dixon ${ }^{2}$ | G.Salles ${ }^{3}$ | A. Wall ${ }^{2}$ | \\ N. Schmitz ${ }^{4}$ | D. Cunningham ${ }^{5}$ | V. Poeschel ${ }^{6}$ | \\ J.Seymour ${ }^{7}$ | U. Jaeger ${ }^{8}$ | T. Habermann ${ }^{9}$ | \\ F. Merli $^{10}$ | C. Haioun ${ }^{11}$ | H. Tilly ${ }^{12}$ | \\ H. Ghesiquieres ${ }^{13}$ | M. Ziepert ${ }^{14}$ | J. Flament ${ }^{15}$ | \\ Q. Shi ${ }^{2}$ | C. Flowers ${ }^{16}$ \\ ${ }^{1}$ Industrial and Systems Engineering, Georgia Institute of Technology, \\ Atlanta, GA, United States; ${ }^{2}$ Department of Health Sciences Research, \\ Mayo Clinic, Rochester, MN, United States; ${ }^{3}$ Hematology, Hospices Civils
}

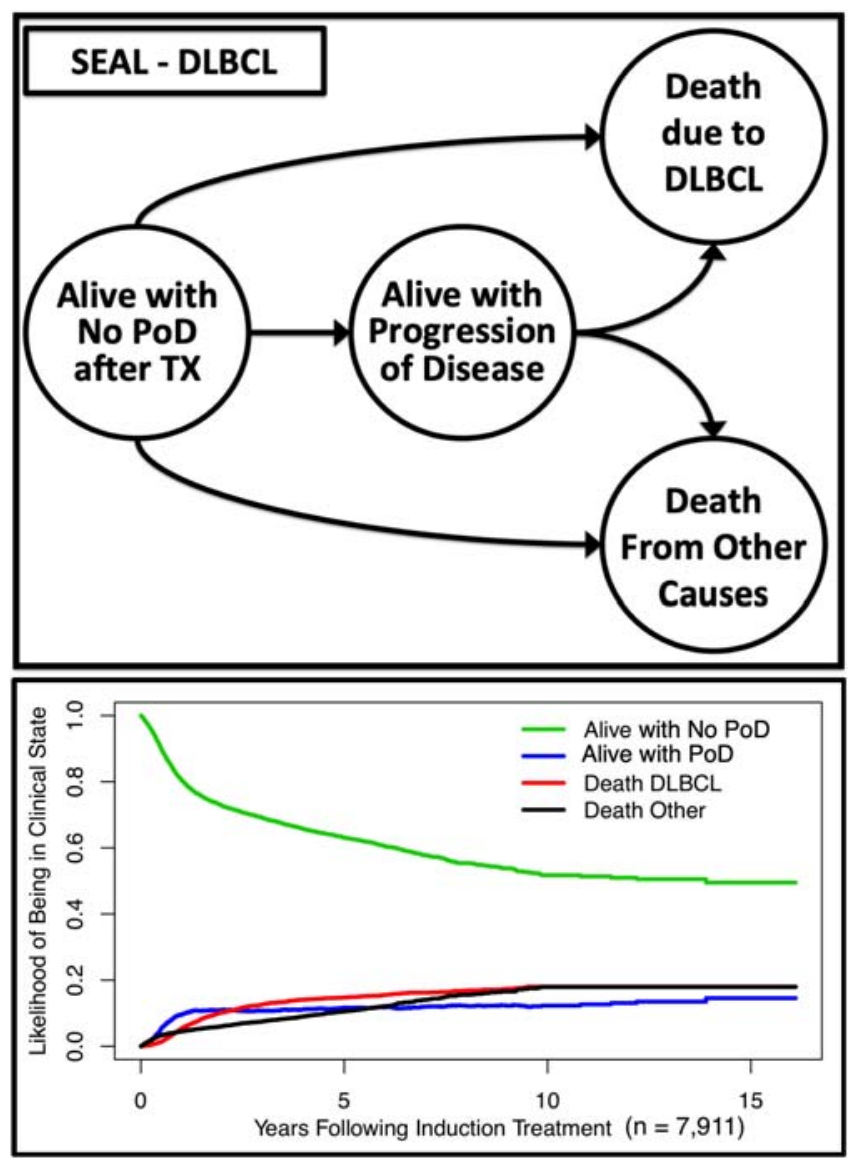

de Lyon, Lyon, France; ${ }^{4}$ Hematology and Oncology, University Hospital Muenster, Muenster, Germany; ${ }^{5}$ Department of Medicine, The Royal Marsden Hospital, Sutton, United Kingdom; ${ }^{6}$ Medical School, Saarland University, Homburg, Germany; ${ }^{7}$ Clinical Research, Integrated Haematology, Peter MacCallum Cancer Centre, Melbourne, Australia; ${ }^{8}$ Department of Medicine I, Medical University of Vienna, Vienna, Austria; ${ }^{9}$ Cancer Center, Hematology, Mayo Clinic, Rochester, MN, United States; ${ }^{10}$ Hematology, AUSL-IRCCS, Reggio Emilia, Italy; ${ }^{11}$ Unite Hemopathies Lymphoides, Hopital Henri Mondor, Creteil, France;

${ }^{12}$ Hématologie, Centre Henri-Becquerel, Rouen, France; ${ }^{13}$ Service d'Hématologie, Centre Hospitalier Lyon-Sud, Pierre Bénite CEDEX, France $;{ }^{14}$ Institut für Medizinische Informatik, Statistik und Epidemiologie, Universität Leipzig, Leipzig, Germany; ${ }^{15}$ Medicine and Biology, Celgene Corporation, Boudry, Switzerland; ${ }^{16}$ Winship Cancer Institute, Emory University, Atlanta, GA, United States

Introduction: The Surrogate Endpoints for Aggressive Lymphoma (SEAL) collaboration established a meta-database integrating individual patient data from 13 first-line randomized clinical trials on previously untreated diffuse large B-cell lymphoma (DLBCL). This metadatabase provides an opportunity to investigate the impact of clinical factors and treatment response on DLBCL-specific and other causes of death following initial therapy to define new opportunities for trials and improve survivorship.

Methods: Using the SEAL database, we studied DLBCL outcomes for patients receiving anthracycline-based chemotherapy with/without added rituximab ( $\mathrm{R}$-Chemo) in a multistate survival analysis model having the model states "Alive with No Progression of Disease (PoD) after Treatment (TX)", "Alive after PoD", "Death from DLBCL", and "Death from Other Causes" (top Figure). The Aalen-Johansen estimator was used to calculate the likelihood of being in each model state and project the course of DLBCL over time (bottom Figure).

Results: We identified 7,911 DLBCL patients (Figure) with median age 62 (range 18-92) years, 62.1\% stage III/IV, 64.7\% IPI $\geq 2$, including 5,108 treated with R-Chemo. Following initial TX for all patients (or with R- Chemo), 2- and 5-year DLBCL-specific death rate were 7.5\% (5.9\%) and 8.8\% (7.0\%) for patients age < 40 at initial TX, 8.9\% (7.2\%) and $12.8 \%(10.6 \%)$ for age $40-60,10.4 \%$ (9.8\%) and $15.2 \%$ (14.6\%) for age $60-70$, and $12.8 \%(12.5 \%)$ and $18.3 \%$ (17.6\%) for age $\geq 70$. Death rates at 2 - and 5 -year from all other causes were $2.3 \%$ (2.1\%) and 3.5\% (3.8\%) for age < 40, 4\% (3.1\%) and 6.5\% (5.1\%) for age $40-60,5.1 \%(4.8 \%)$ and $9.4 \%(7.9 \%)$ for age $60-70$, and $11.3 \%$ (12.3\%) and $18.3 \%(20.0 \%)$ for age $\geq 70$. Following R- Chemo ( $n=5,108$ ), 2-year PoD rates were $12.3 \%$ for patients achieving $C R$, 24.1\% for PR, and $28.4 \%$ for SD; 2 - and 5- year DLBCL-specific mortality rates were $4.6 \%$ and $8.7 \%$ for CR, $11.8 \%$ and $18.6 \%$ for PR, $20.3 \%$ and $25.6 \%$ for SD, and $69.1 \%$ and $71.2 \%$ for PD. These rates were similar for "non R-Chemo" regimens. International Prognostic Index (IPI) affected the 2- and 5-year DLBCL-specific mortality rates, which were $4.8 \%$ and $8 \%$ for IPI $=0$ or $1,7.8 \%$ and $11.9 \%$ for IPI $=$ $2,13.3 \%$ and $18.6 \%$ for IPI $=3$, and $24.4 \%$ and $29.7 \%$ for IPI $=4$ or 5 in the whole SEAL population $(n=7,911)$. 